\title{
The Amalgamated Duplication of a Ring Along a Semidualizing Ideal
}

\author{
Maryam Salimi $(*)$ - Elham Tavasoli $(* *)$ - Siamak Yassemi $(* * *)$
}

ABstRact - Let $R$ be a commutative Noetherian ring and let $I$ be an ideal of $R$. In this paper, after recalling briefly the main properties of the amalgamated duplication ring $R \bowtie I$ which is introduced by D'Anna and Fontana, we restrict our attention to the study of the properties of $R \bowtie I$, when $I$ is a semidualizing ideal of $R$, i.e., $I$ is an ideal of $R$ and $I$ is a semidualizing $R$-module. In particular, it is shown that if $I$ is a semidualizing ideal and $M$ is a finitely generated $R$-module, then $M$ is totally $I$-reflexive as an $R$-module if and only if $M$ is totally reflexive as an $(R \bowtie I)$-module. In addition, it is shown that if $I$ is a semidualizing ideal, then $R$ and $I$ are Gorenstein projective over $R \bowtie I$, and every injective $R$-module is Gorenstein injective as an $(R \bowtie I)$-module. Finally, it is proved that if $I$ is a non-zero flat ideal of $R$, then $\operatorname{fd}_{R}(M)=\mathrm{fd}_{R \bowtie I}\left(M \otimes_{R}(R \bowtie I)\right)=\mathrm{fd}_{R}\left(M \otimes_{R}(R \bowtie I)\right)$, for every $R$-module $M$.

Mathematics Subject Classification (2010). 13D05, $13 \mathrm{H} 10$.

KEYWORDS. Amalgamated duplication, semidualizing, totally reflexive, Gorenstein projective.

\section{Introduction}

Throughout this paper all rings are considered commutative with identity element, and all ring homomorphisms are unital. Over a commutative local ring, semidualizing modules provide a common generalization

(*) Indirizzo dell'A.: Department of Mathematics, Science and Research Branch, Islamic Azad University, Tehran, Iran.

E-mail: maryamsalimi@ipm.ir

(**) Indirizzo dell'A.: Department of Mathematics, Science and Research Branch, Islamic Azad University, Tehran, Iran.

E-mail: elhamtavasoli@ipm.ir

(***) Indirizzo dell'A.: Department of Mathematics, University of Tehran, and School of mathematics, Institute for research in fundamental sciences (IPM), Tehran, Iran.

E-mail: yassemi@ipm.ir

The research of Siamak Yassemi was in part supported by a grant from IPM (No. 91130214). 
of a dualizing (canonical) module and a free module of rank one. In [10], Golod introduced the notion of semidualizing modules, though suggestions of this notion can be traced back to Foxby [9] and Vasconcelos [18].

Let $R$ be a ring and let $M$ be an $R$-module. The idealization $R \times M$ (also called trivial extension) introduced by Nagata in 1956 [14, Page 2], is a new ring where the module $M$ can be viewed as an ideal such that its square is 0 . In [11, Lemma 3.1], it is proved that if $C$ is a semidualizing module for Noetherian ring $R$, then every injective $(R \times C)$-module is a direct summand of the $R$-module $\operatorname{Hom}_{R}(R \times C, E)$, where $E$ is an injective $R$-module. Also, Holm and Jørgensen in [11, Lemma 3.3], showed that if $C$ is a semidualizing module for Noetherian ring $R$, then

(i) $R$ and $C$ are Gorenstein projective over $R \times C$.

(ii) $\operatorname{Hom}_{R}(R, E) \cong E$ and $\operatorname{Hom}_{R}(C, E)$ are Gorenstein injective over $R \times C$.

This paper builds on work of Holm and Jørgensen for the amalgamated duplication instead of idealization. First, we recall the definition of amalgamated duplication. In [6] D'Anna and Fontana, considered a different type of construction obtained involving a ring $R$ and an ideal $I \subset R$ that is denoted by $R \bowtie I$, called amalgamated duplication, and it is defined as the following subring of $R \times R$ :

$$
R \bowtie I=\{(r, r+i) \mid r \in R, i \in I\} .
$$

They showed that $R \bowtie I$ is reduced if and only if $R$ is reduced. This construction has been studied by different authors, since it allows to produce rings satisfying preassigned conditions and this is the main motivation for the investigations of the authors. In this paper, we study the ring $R \bowtie I$ for semidualizing ideal $I$. In Section 3, we show that if $I$ is an ideal of Noetherian ring $R$ such that $I$ is a semidualizing $R$-module, and $M$ is a finitely generated $R$-module, then $M$ is totally $I$-reflexive as an $R$-module if and only if $M$ is totally reflexive as an $(R \bowtie I)$-module (see Theorem 3.2). Also, we show that, if $I$ is a non-zero ideal of Noetherian ring $R$ such that $I$ is a semidualizing $R$-module and $M$ is a totally reflexive $(R \bowtie I)$-module, then

$$
\operatorname{Tor}_{i}^{R}(M, N)=0=\operatorname{Ext}_{R}^{i}\left(M, I \otimes_{R} N\right)
$$

for all $i \geq 1$, provided that $N$ is a locally finite flat dimension $R$-module, and

$$
\operatorname{Ext}_{R}^{i}(M, G)=0=\operatorname{Tor}_{i}^{R}\left(M, \operatorname{Hom}_{R}(I, G)\right)
$$

for all $i \geq 1$, provided that $G$ is a locally finite injective dimension $R$ module. 
Finally, we prove that if $I$ is an ideal of Noetherian ring $R$, then every injective $(R \bowtie I)$-module is a direct summand of the $R$-module $\operatorname{Hom}_{R}(R \bowtie I, M)$, where $M$ is an injective $R$-module. In addition, we show that if $I$ is semidualizing, then $R$ and $I$ are Gorenstein projective over $R \bowtie I$, and every injective $R$-module is Gorenstein injective as an $(R \bowtie I)$-module.

\section{Preliminaries}

First, we deal with some applications of a general construction, introduced in [6], called amalgamated duplication of a ring along an ideal.

Let $R$ be a commutative ring with unit element 1 and let $I$ be an ideal of $R$. Set

$$
R \bowtie I=\{(r, s) \mid r, s \in R, s-r \in I\} .
$$

It is easy to check that $R \bowtie I$ is a subring, with unit element $(1,1)$, of $R \times R$ (with the usual componentwise operations) and that $R \bowtie I=$ $\{(r, r+i) \mid r \in R, i \in I\}$. In the following, we recall some main properties of the ring $R \bowtie I$ from [5] which we use in the next section.

Proposition 2.1. Let $R$ be a ring and let $I$ be an ideal of $R$. Then the following statements hold.

(i) By introducing a multiplicative structure in the R-module direct sum $R \oplus I$ by setting

$$
(r, i)(s, j)=(r s, r j+s i+i j),
$$

the map $f: R \oplus I \rightarrow R \bowtie I$ defined by $f((r, i))=(r, r+i)$ is a ring isomorphism and $R$-isomorphism too. Moreover, there is an split exact sequence of $R$-modules

$$
0 \rightarrow R \stackrel{\varphi}{\rightarrow} R \bowtie I \stackrel{\psi}{\rightarrow} I \rightarrow 0
$$

where $\varphi(r)=(r, r)$ for all $r \in R$, and $\psi((r, s))=s-r$, for all $(r, s) \in R \bowtie I$. Notice that this sequence splits.

(ii) $R$ and $R \bowtie I$ have the same Krull dimension. Also, if $R$ is a Noetherian ring, then $R \bowtie I$ is a finitely generated $R$-module.

In [1], [5], [6], [16], and [17], the properties of the ring $R \bowtie I$ were studied extensively.

The following notion was introduced by Golod, though suggestion of this notion can be traced back to Foxby and Vasconcelos. 
Definition 2.2. Let $C$ be a finitely generated $R$-module. An $R$-module $G$ is totally $C$-reflexive if it satisfies the following conditions:

(i) $G$ is finitely generated over $R$;

(ii) the biduality map $\delta_{G}^{C}: G \longrightarrow \operatorname{Hom}_{R}\left(\operatorname{Hom}_{R}(G, C), C\right)$ given by $\delta_{G}^{C}(g)(\psi)=\psi(g)$ for each $g \in G$ and $\psi \in \operatorname{Hom}_{R}(G, C)$, is an isomorphism; and

(iii) $\operatorname{Ext}_{R}^{i}(G, C)=0=\operatorname{Ext}_{R}^{i}\left(\operatorname{Hom}_{R}(G, C), C\right)$ for all $i \geq 1$.

In this paper, for an $R$-module $M$, we use " $M$ is totally reflexive" instead of " $M$ is totally $R$-reflexive".

The following notion was introduced, seemingly independently and using different terminology, by Foxby, Golod, Wakamatsu, and Vasconcelos.

Definition 2.3. The $R$-module $C$ is semidualizing if it satisfies the following:

(i) $C$ is finitely generated;

(ii) the homothety map $\chi_{C}^{R}: R \longrightarrow \operatorname{Hom}_{R}(C, C)$ given by $\chi_{C}^{R}(r)(c)=r c$ for each $r \in R$ and $c \in C$, is an isomorphism; and

(iii) $\operatorname{Ext}_{R}^{i}(C, C)=0$ for all $i \geq 1$.

In addition an $R$-module $D$ is called dualizing if it is semidualizing and $\operatorname{id}_{R}(D)<\infty$.

By the term " $I$ is a semidualizing ideal for ring $R$ ", we mean that $I$ is an ideal of $R$ and $I$ is a semidualizing $R$-module.

Recall that a complete projective resolution is an exact sequence of projective modules

$$
\boldsymbol{P}: \cdots \longrightarrow P_{1} \longrightarrow P_{0} \longrightarrow P^{0} \longrightarrow P^{1} \longrightarrow \cdots,
$$

such that $\operatorname{Hom}_{R}(\boldsymbol{P}, Q)$ is exact for every projective $R$-module $Q$. An $R$ module $M$ is called Gorenstein projective, if there exists a complete projective resolution $\boldsymbol{P}$ with $M \cong \operatorname{coker}\left(P_{0} \longrightarrow P^{0}\right)$. The notion of Gorenstein injective modules are defined dually.

\section{Main Results}

Throughout this section, $R$ is a Noetherian ring. We follow standard notation and terminology from [2]. 
In this section, we study the ring $R \bowtie I$ for a semidualizing ideal $I$. In the following Theorem, we show that a finitely generated $R$-module $M$ is totally $I$-reflexive if and only if $M$ is totally reflexive as an $(R \bowtie I)$-module, provided that $I$ is a semidualizing $R$-module. First, we prove the following lemma:

Lemma 3.1. Let I be a semidualizing ideal of $R$. Then the following statements hold.

(i) There is an $(R \bowtie I)$-isomorphism $\operatorname{Hom}_{R}(R \bowtie I, I) \cong R \bowtie I$.

(ii) There is a natural equivalence of $R$-modules

$$
\operatorname{Hom}_{R \bowtie I}(-, R \bowtie I) \cong \operatorname{Hom}_{R}(-, I) .
$$

(iii) Let $M$ be an $R$-module. Then the biduality map

$$
M \longrightarrow \operatorname{Hom}_{R}\left(\operatorname{Hom}_{R}(M, I), I\right)
$$

is an R-isomorphism if and only if the biduality map

$$
M \longrightarrow \operatorname{Hom}_{R \bowtie I}\left(\operatorname{Hom}_{R \bowtie I}(M, R \bowtie I), R \bowtie I\right)
$$

is an $(R \bowtie I)$-isomorphism.

(iv) There is an $(R \bowtie I)$-isomorphism $\operatorname{Hom}_{R \bowtie I}(R, R \bowtie I) \cong I$.

(v) If $E$ is an injective $R$-module, then there is an equivalence of $(R \bowtie I)$-module

$$
\operatorname{Hom}_{R \bowtie I}\left(\operatorname{Hom}_{R}(R \bowtie I, E),-\right) \cong \operatorname{Hom}_{R}\left(\operatorname{Hom}_{R}(I, E),-\right) .
$$

Proof. (i) We have the following isomorphisms of $R$-modules:

$$
\begin{aligned}
\operatorname{Hom}_{R}(R \bowtie I, I) \cong \operatorname{Hom}_{R}(R \oplus I, I) & \cong \operatorname{Hom}_{R}(I, I) \oplus \operatorname{Hom}_{R}(R, I) \\
& \cong R \oplus I \cong R \bowtie I,
\end{aligned}
$$

since $I$ is semidualizing. The proof of [1, Theorem 3.3] implies that the $R$ isomorphism $\operatorname{Hom}_{R}(R \bowtie I, I) \cong R \bowtie I$ is an $(R \bowtie I)$-isomorphism.

(ii) Let $M$ be an $R$-module. In the following sequence, the first isomorphism is from (i), the second isomorphism is from Hom-tensor adjointness, and the third isomorphism is induced by tensor cancellation:

$$
\begin{aligned}
\operatorname{Hom}_{R \bowtie I}(M, R \bowtie I) & \cong \operatorname{Hom}_{R \bowtie I}\left(M, \operatorname{Hom}_{R}(R \bowtie I, I)\right) \\
& \cong \operatorname{Hom}_{R}\left(M \otimes_{R \bowtie I}(R \bowtie I), I\right) \\
& \cong \operatorname{Hom}_{R}(M, I) .
\end{aligned}
$$


(iii) In the next sequence, the first isomorphism is from (i), (ii) and Hom-tensor adjointness, and the second isomorphism is induced by tensor cancellation:

$$
\begin{aligned}
\operatorname{Hom}_{R \bowtie I}\left(\operatorname{Hom}_{R \bowtie I}(M, R \bowtie I), R \bowtie I\right) & \cong \operatorname{Hom}_{R}\left(\operatorname{Hom}_{R}(M, I) \otimes_{R \bowtie I}(R \bowtie I), I\right) \\
& \cong \operatorname{Hom}_{R}\left(\operatorname{Hom}_{R}(M, I), I\right) .
\end{aligned}
$$

It shows that there is an $(R \bowtie I)$-isomorphism

$$
M \cong \operatorname{Hom}_{R \bowtie I}\left(\operatorname{Hom}_{R \bowtie I}(M, R \bowtie I), R \bowtie I\right)
$$

if and only if there is an $R$-isomorphism

$$
M \cong \operatorname{Hom}_{R}\left(\operatorname{Hom}_{R}(M, I), I\right) .
$$

By [15, Proposition 5.4.1 (a)], the biduality map $\delta_{M}^{R \bowtie I}$ is an $(R \bowtie I)$-isomorphism if and only if the biduality map $\delta_{M}^{I}$ is an $R$-isomorphism.

(iv) It follows from $(i i)$. Note that $I$ has $(R \bowtie I)$-module structure by Proposition $2.1(i)$.

$(v)$ In the following sequence, the first isomorphism follows from $(i)$, the second isomorphism follows from [7, Thorem 3.2.11], and the third isomorphism is induced by Hom-tensor adjointness:

$$
\begin{aligned}
\operatorname{Hom}_{R \bowtie I}\left(\operatorname{Hom}_{R}(R \bowtie I, E),-\right) & \cong \operatorname{Hom}_{R \bowtie I}\left(\operatorname{Hom}_{R}\left(\operatorname{Hom}_{R}(R \bowtie I, I), E\right),-\right) \\
& \cong \operatorname{Hom}_{R \bowtie I}\left(\operatorname{Hom}_{R}(I, E) \otimes_{R}(R \bowtie I),-\right) \\
& \cong \operatorname{Hom}_{R}\left(\operatorname{Hom}_{R}(I, E),-\right) .
\end{aligned}
$$

THEOREM 3.2. Let $I$ be a semidualizing ideal of $R$, and let $M$ be a finitely generated $R$-module. Then $M$ is totally $I$-reflexive as an $R$-module if and only if $M$ is totally reflexive as an $(R \bowtie I)$-module.

Proof. Let $E^{\bullet}$ be an injective resolution of $I$ as an $R$-module. Let $\varphi: R \rightarrow R \bowtie I$ be $R$-homomorphism in Proposition 2.1(i). By [15, Propositions 2.1.12 and 2.1.13], $I$ and $R$ are totally $I$-reflexive, so according to [15, Proposition 2.1.4], $R \oplus I$ is totally $I$-reflexive. Therefore, $R \bowtie I$ is totally $I$ reflexive by Proposition 2.1(i). Hence $\operatorname{Ext}_{R}^{i}(R \bowtie I, I)=0$ for all $i \geq 1$, so the complex $\operatorname{Hom}_{R}\left(R \bowtie I, E^{\bullet}\right)$ is an injective resolution of the $(R \bowtie I)$ module $\operatorname{Hom}_{R}(R \bowtie I, I)$. By Lemma 3.1(i), $\operatorname{Hom}_{R}\left(R \bowtie I, E^{\bullet}\right)$ is an injective resolution of the $(R \bowtie I)$-module $R \bowtie I$. This yields the first isomorphism in the next sequence: 


$$
\begin{aligned}
\operatorname{Ext}_{R \bowtie I}^{i}(M, R \bowtie I) & \cong \mathrm{H}_{-i}\left(\operatorname{Hom}_{R \bowtie I}\left(M, \operatorname{Hom}_{R}\left(R \bowtie I, E^{\bullet}\right)\right)\right) \\
& \cong \mathrm{H}_{-i}\left(\operatorname{Hom}_{R}\left((R \bowtie I) \otimes_{R \bowtie I} M, E^{\bullet}\right)\right) \\
& \cong \mathrm{H}_{-i}\left(\operatorname{Hom}_{R}\left(M, E^{\bullet}\right)\right) \\
& \cong \operatorname{Ext}_{R}^{i}(M, I) .
\end{aligned}
$$

It follows that $\operatorname{Ext}_{R \bowtie I}^{i}(M, R \bowtie I)=0$ for all $i \geq 1$ if and only if $\operatorname{Ext}_{R}^{i}(M, I)=0$ for all $i \geq 1$. Also, we have the following isomorphisms:

$$
\begin{aligned}
\operatorname{Ext}_{R \bowtie I}^{i}\left(\operatorname{Hom}_{R \bowtie I}(M, R \bowtie I), R \bowtie I\right) & \cong \operatorname{Ext}_{R \bowtie I}^{i}\left(\operatorname{Hom}_{R}(M, I), R \bowtie I\right) \\
& \cong \operatorname{Ext}_{R}^{i}\left(\operatorname{Hom}_{R}(M, I), I\right) .
\end{aligned}
$$

In the above sequence, the first isomorphism follows from Lemma 3.1(ii) and the second one follows from (*), using $\operatorname{Hom}_{R}(M, I)$ in place of $M$. It follows that $\operatorname{Ext}_{R \bowtie I}^{i}\left(\operatorname{Hom}_{R \bowtie I}(M, R \bowtie I), R \bowtie I\right)=0$ for all $i \geq 1$ if and only if $\operatorname{Ext}_{R}^{i}\left(\operatorname{Hom}_{R}(M, I), I\right)=0$ for all $i \geq 1$. By Lemma 3.1(iii), the biduality map $M \longrightarrow \operatorname{Hom}_{R}\left(\operatorname{Hom}_{R}(M, I), I\right)$ is an isomorphism if and only if the biduality map $M \longrightarrow \operatorname{Hom}_{R \bowtie I}\left(\operatorname{Hom}_{R \bowtie I}(M, R \bowtie I), R \bowtie I\right)$ is an isomorphism. So, the assertion follows from definition.

Corollary 3.3. Let I be a semidualizing ideal of $R$. Then I is totally reflexive as an $(R \bowtie I)$-module.

Proof. By [15, Proposition 2.1.12], $I$ is totally $I$-reflexive as an $R$ module. So by Theorem 3.2, $I$ is totally reflexive as an $(R \bowtie I)$-module.

Corollary 3.4. Let I be a non-zero semidualizing ideal of $R$, and let $M$ be a totally reflexive $(R \bowtie I)$-module. Then the following statements hold.

(i) If $N$ is a locally finite flat dimension $R$-module, then

$$
\operatorname{Tor}_{i}^{R}(M, N)=0=\operatorname{Ext}_{R}^{i}\left(M, I \otimes_{R} N\right)
$$

for all $i \geq 1$.

(ii) If $N$ is a locally finite injective dimension $R$-module, then

$$
\operatorname{Ext}_{R}^{i}(M, N)=0=\operatorname{Tor}_{i}^{R}\left(M, \operatorname{Hom}_{R}(I, N)\right)
$$

for all $i \geq 1$. 
Proof. By Theorem 3.2, $M$ is totally $I$-reflexive as an $R$-module. Therefore, $(i)$ follows from [15, Proposition 5.4.8], and (ii) follows from [15, Proposition 5.4.9].

In [15], Sather-Wagstaff investigated the behavior of semidualizing and totally reflexive modules under flat based change of ring, indeed, in [15, Propositions 2.2.1 and 5.3.1], he showed that if $\varphi: R \longrightarrow S$ is a flat ring homomorphism, then the following statements hold.

(i) Let $C$ be a finitely generated $R$-module. If $C$ is a semidualizing $R$-module, then $C \otimes_{R} S$ is a semidualizing $S$-module. The converse holds when $\varphi$ is faithfully flat.

(ii) Let $C$ be a semidualizing $R$-module and let $M$ be a finitely generated $R$-module. If $M$ is totally $C$-reflexive, then the $S$ module $C \otimes_{R} M$ is totally $\left(C \otimes_{R} S\right)$-reflexive. The converse holds when $\varphi$ is faithfully flat.

Assume that $I$ is a non-zero flat ideal of Noetherian ring $R$. It is straightforward to see that the map $\varphi: R \longrightarrow R \bowtie I$ is faithfully flat, since $R \bowtie I \cong R \oplus I$, by Proposition 2.1(i). So we have:

Lemma 3.5. Let I be a non-zero flat ideal of $R$. Then the following statements hold:

(i) Let $C$ be a finitely generated $R$-module. Then $C$ is semidualizing $R$-module if and only if $C \otimes_{R}(R \bowtie I)$ is a semidualizing $(R \bowtie I)$-module.

(ii) Let $C$ be a semidualizing $R$-module and let $M$ be a finitely generated $R$-module. Then $M$ is totally $C$-reflexive if and only if the $(R \bowtie I)$-module $M \otimes_{R}(R \bowtie I)$ is totally $\left(C \otimes_{R}(R \bowtie I)\right)$-reflexive.

Proposition 3.6. Assume that I is a non-zero flat ideal of $R$. Let $C$ be a semidualizing $R$-module, and let $M$ be a totally $C$-reflexive $R$-module. Then the following statements hold.

(i) If $N$ is a locally finite flat dimension $(R \bowtie I)$-module, then

$$
\operatorname{Tor}_{i}^{R \bowtie I}(M, N)=0=\operatorname{Ext}_{R \bowtie I}^{i}\left(M \otimes_{R}(R \bowtie I), C \otimes_{R} N\right)
$$

for all $i \geq 1$.

(ii) If $N$ is a locally finite injective dimension $(R \bowtie I)$-module, then $\operatorname{Tor}_{i}^{R \bowtie I}\left(M, \operatorname{Hom}_{R \bowtie I}\left(C \otimes_{R}(R \bowtie I), N\right)\right)=0=\operatorname{Ext}_{R \bowtie I}^{i}\left(M \otimes_{R}(R \bowtie I), N\right)$ for all $i \geq 1$. 
Proof. Note that $R \bowtie I$ is a flat $R$-module, since $I$ is a flat ideal of $R$. By Lemma 3.5, $C \otimes_{R}(R \bowtie I)$ is a semidualizing $(R \bowtie I)$-module and $M \otimes_{R}(R \bowtie I)$ is a totally $\left(C \otimes_{R}(R \bowtie I)\right)$-reflexive $(R \bowtie I)$-module.

(i) In the following sequence, the equality follows from [15, Proposition 5.4.8], and the isomorphism holds, since $R \bowtie I$ is a flat $R$-module

$$
\begin{aligned}
0 & =\operatorname{Tor}_{i}^{R \bowtie I}\left(M \otimes_{R}(R \bowtie I), N\right) \\
& \cong \operatorname{Tor}_{i}^{R \bowtie I}(M, N) \otimes_{R}(R \bowtie I),
\end{aligned}
$$

for all $i \geq 1$. So, $\operatorname{Tor}_{i}^{R \bowtie I}(M, N)=0$, since $R \bowtie I$ is faithfully flat $R$ module.

In the following sequence, for all $i \geq 1$, the equality follows from [15, Proposition 5.4.8], and the isomorphism induces by tensor cancellation:

$$
\begin{aligned}
0 & =\operatorname{Ext}_{R \bowtie I}^{i}\left(M \otimes_{R}(R \bowtie I),\left(C \otimes_{R}(R \bowtie I)\right) \otimes_{R \bowtie I} N\right) \\
& \cong \operatorname{Ext}_{R \bowtie I}^{i}\left(M \otimes_{R}(R \bowtie I), C \otimes_{R} N\right) .
\end{aligned}
$$

(ii) In the following sequence, for all $i \geq 1$, the equality holds by [15, Proposition 5.4.9], and the isomorphism holds, since $R \bowtie I$ is a flat $R$-module

$$
\begin{aligned}
0 & =\operatorname{Tor}_{i}^{R \bowtie I}\left(M \otimes_{R}(R \bowtie I), \operatorname{Hom}_{R \bowtie I}\left(C \otimes_{R}(R \bowtie I), N\right)\right) \\
& \cong \operatorname{Tor}_{i}^{R \bowtie I}\left(M, \operatorname{Hom}_{R \bowtie I}\left(C \otimes_{R}(R \bowtie I), N\right)\right) \otimes_{R}(R \bowtie I) .
\end{aligned}
$$

So, $\operatorname{Tor}_{i}^{R \bowtie I}\left(M, \operatorname{Hom}_{R \bowtie I}\left(C \otimes_{R}(R \bowtie I), N\right)\right)=0$, since $R \bowtie I$ is a faithfully flat $R$-module. By [15, Proposition 5.4.9], we have

$$
\operatorname{Ext}_{R \bowtie I}^{i}\left(M \otimes_{R}(R \bowtie I), N\right)=0 .
$$

Let $I$ be a semidualizing ideal of $R$. In the following, we investigate that $R$ and $I$ are Gorenstein projective over $R \bowtie I$. Also, we show that every injective $R$-module is Gorenstein injective as an $(R \bowtie I)$-module. First, we prove the following lemma:

Lemma 3.7. Let I be an ideal of $R$. Then the following statements hold.

(i) If $M$ is a (faithfully) injective $R$-module, then $\operatorname{Hom}_{R}(R \bowtie I, M)$ is a (faithfully) injective $(R \bowtie I)$-module.

(ii) Every injective $(R \bowtie I)$-module is a direct summand of the $R$-module $\operatorname{Hom}_{R}(R \bowtie I, M)$, where $M$ is an injective $R$-module. 
Proof. (i) The following sequence of $(R \bowtie I)$-isomorphisms makes clear that if $M$ is a (faithfully) injective $R$-module, then $\operatorname{Hom}_{R}(R \bowtie I, M)$ is a (faithfully) injective $(R \bowtie I)$-module

$$
\begin{aligned}
\operatorname{Hom}_{R \bowtie I}\left(-, \operatorname{Hom}_{R}(R \bowtie I, M)\right) & \cong \operatorname{Hom}_{R}\left((R \bowtie I) \otimes_{R \bowtie I}-, M\right) \\
& \cong \operatorname{Hom}_{R}(-, M) .
\end{aligned}
$$

Note that in the above sequence, the first isomorphism follows from Homtensor adjointness, and the second isomorphism is induced by tensor cancellation.

(ii) Let $J$ be an injective ( $R \bowtie I)$-module. It is enough to show that $J$ is embeded into an $R$-module of the form $\operatorname{Hom}_{R}(R \bowtie I, M)$ where $M$ is an injective $R$-module. Consider $J$ as an $R$-module and embed it into an injective $R$-module $M$. Then use isomorphisms in part (i), to convert the monomorphism of $R$-modules $J \hookrightarrow M$ to a monomorphism of $(R \bowtie I)$-modules $J \hookrightarrow \operatorname{Hom}_{R}(R \bowtie I, M)$.

THEOREM 3.8. Let I be a semidualizing ideal of $R$, and let $E$ be an injective R-module. Then the following statements hold.

(i) $R$ and $I$ are Gorenstein projective over $R \bowtie I$.

(ii) $\operatorname{Hom}_{R}(R, E) \cong E$ and $\operatorname{Hom}_{R}(I, E)$ are Gorenstein injective over $R \bowtie I$.

Proof. (i) By Lemma $3.1(i v), \operatorname{Hom}_{R \bowtie I}(R, R \bowtie I) \cong I$. It means, the dual of $R$ with respect to the ring $R \bowtie I$ is $I$. But dualization with respect to the ring preserves the class of finitely generated Gorenstein projective modules by [3, Observation (1.1.7)]. So, to prove part ( $i$ ), it is enough to show that $R$ is Gorenstein projective over $R \bowtie I$. By [4, Proposition 2.16], it is enough to show that $R$ is a totally reflexive $(R \bowtie I)$-module, since $R$ is a Noetherian ring and $R$ is a finitely generated $(R \bowtie I)$-module. By Theorem 3.2 , it suffices to show that $R$ is a totally $I$-reflexive $R$-module, and this follows from [15, Proposition 2.1.13].

(ii) First, we show that $\operatorname{Hom}_{R}(I, E)$ is Gorenstein injective over $R \bowtie I$. Since $I$ is Gorenstein projective over $R \bowtie I$, it has a complete projective resolution as follows:

$$
\boldsymbol{P}: \cdots \longrightarrow P_{1} \stackrel{\partial^{1}}{\longrightarrow} P_{0} \stackrel{\partial^{0}}{\longrightarrow} P_{-1} \longrightarrow \cdots,
$$

such that $P_{i}$ are projective $(R \bowtie I)$-modules and $I \cong$ coker $\partial^{1}$, and $\operatorname{Hom}_{R \bowtie I}(\boldsymbol{P}, Q)$ is exact where $Q$ is a projective $(R \bowtie I)$-module. We can assume that $\boldsymbol{P}$ consists of finitely generated $(R \bowtie I)$-modules, since $R$ is a 
Noetherian ring. By Lemma 3.7(i), the $(R \bowtie I)$-module $J=\operatorname{Hom}_{R}(R \bowtie I, E)$ is injective. Consider the exact complex $\boldsymbol{K}=\operatorname{Hom}_{R \bowtie I}(\boldsymbol{P}, J)$ of injective $(R \bowtie I)$-modules. Note that for every injective $(R \bowtie I)$-module $L$ we have

$$
\begin{aligned}
\operatorname{Hom}_{R \bowtie I}(L, \boldsymbol{K}) & =\operatorname{Hom}_{R \bowtie I}\left(L, \operatorname{Hom}_{R \bowtie I}(\boldsymbol{P}, J)\right) \\
& \cong \operatorname{Hom}_{R \bowtie I}\left(L \otimes_{R \bowtie I} \boldsymbol{P}, J\right) \\
& \cong \operatorname{Hom}_{R \bowtie I}\left(\boldsymbol{P}, \operatorname{Hom}_{R \bowtie I}(L, J)\right) .
\end{aligned}
$$

Also, $\operatorname{Hom}_{R \bowtie I}(L, J)$ is a flat $(R \bowtie I)$-module, so it is the direct limit of projective $(R \bowtie I)$-modules $Q_{\alpha}$, that is, $\operatorname{Hom}_{R \bowtie I}(L, J) \cong \lim _{\longrightarrow} Q_{\alpha}$. So by (*)

$$
\operatorname{Hom}_{R \bowtie I}(L, \boldsymbol{K}) \cong \operatorname{Hom}_{R \bowtie I}\left(\boldsymbol{P}, \lim _{\longrightarrow} Q_{\alpha}\right) \cong \lim \operatorname{Hom}_{R \bowtie I}\left(\boldsymbol{P}, Q_{\alpha}\right),
$$

is exact. This shows that $\boldsymbol{K}$ is a complete injective resolution over $R \bowtie I$ and

$$
\begin{aligned}
\operatorname{ker}\left(\operatorname{Hom}_{R \bowtie I}\left(\partial^{1}, J\right)\right) & =\operatorname{Hom}_{R \bowtie I}\left(\operatorname{coker}\left(\partial^{1}\right), J\right) \\
& \cong \operatorname{Hom}_{R \bowtie I}\left(I, \operatorname{Hom}_{R}(R \bowtie I, E)\right) \\
& \cong \operatorname{Hom}_{R}\left(I \otimes_{R \bowtie I}(R \bowtie I), E\right) \\
& \cong \operatorname{Hom}_{R}(I, E) .
\end{aligned}
$$

Therefore, $\operatorname{Hom}_{R}(I, E)$ is a Gorenstein injective $(R \bowtie I)$-module.

Using the same argument, one can show that $\operatorname{Hom}_{R}(R, E) \cong E$ is Gorenstein injective over $R \bowtie I$.

In [12] and [13], some properties of homological dimension of $R \bowtie I$ were investigated.

Proposition 3.9. Let I be a non-zero semidualizing ideal of local ring R. Then $I$ is a dualizing R-module if and only if $R \bowtie I$ is Gorenstein.

Proof. By [13, Lemma 2.1], $\operatorname{id}_{R \bowtie I}(R \bowtie I)=\operatorname{id}_{R}(I)$. So, the assertion follows by definition.

COROLlary 3.10. Let I be a non-zero semidualizing ideal of local ring $R$. Then $I$ is an injective $R$-module if and only if $R \bowtie I$ is a quasiFrobenius ring.

Proof. Recall that the ring $R$ is called quasi-Frobenius if $R$ is a Gorenstein ring of Krull dimension zero (see [7]). The assertion follows from Proposition 3.9. 
Proposition 3.11. Let I be a non-zero flat ideal of $R$. For every $R$ module $M$ we have:

(i) $\operatorname{id}_{R}(M)=\operatorname{id}_{R}\left(M \otimes_{R}(R \bowtie I)\right)$

(ii) $\operatorname{fd}_{R}(M)=\operatorname{fd}_{R}\left(M \otimes_{R}(R \bowtie I)\right)$

Proof. Note that $R \bowtie I$ is a faithfully flat $R$-module. (i) follows from [20, Corollary 2.9] and (ii) follows from [20, Corollary 2.11].

COROLLARY 3.12. Let I be a non-zeroflatideal of R. Forevery R-module $M$, we have

$$
\operatorname{fd}_{R}(M)=\operatorname{fd}_{R \bowtie I}\left(M \otimes_{R}(R \bowtie I)\right)=\operatorname{fd}_{R}\left(M \otimes_{R}(R \bowtie I)\right) .
$$

Proof. By [12, Lemma 2.3], we have $\operatorname{fd}_{R}(M)=\operatorname{fd}_{R \bowtie I}\left(M \otimes_{R}(R \bowtie I)\right)$, and by Proposition 3.11, $\mathrm{fd}_{R}(M)=\mathrm{fd}_{R}\left(M \otimes_{R}(R \bowtie I)\right)$.

Proposition 3.13. Let $I$ be a non-zero ideal of a ring $R$ of Krull dimension zero. If $R \bowtie I$ is a Gorenstein ring, then $\operatorname{id}_{R}(R \bowtie I)=$ $\mathrm{fd}_{R}(R \bowtie I)$.

Proof. Note that $\operatorname{dim}(R \bowtie I)=\operatorname{dim}(R)=0$, so $R \bowtie I$ is self-injective. By [20, Corollary 3.4], $\operatorname{id}_{R}(R \bowtie I)=\mathrm{fd}_{R}(R \bowtie I)$.

Theorem 3.14. Let I be an ideal of Artinian ring $R$. Then $R$ is Gorenstein if and only if $\operatorname{Ext}_{R}^{i}(k, R)=0$.

Proof. By assumption, $R$ is Gorenstein if and only if $R$ is self injective. Note that $R$ is an Artinian ring if and only if $R$ is an Artinian $(R \bowtie I)$-module. So, the assertion follows [8, Theorem 6.1].

Acknowledgments. The authors are very grateful to the referee for several comments that greatly improved the paper.

\section{REFERENCES}

[1] A. Bagheri - M. Salimi - E. Tavasoli - S. Yassemi, A Construction of QuasiGorenstein Rings, J. Algebra Appl. 11, no. 1 (2012), 1250013 (9 pages).

[2] W. Bruns - J. Herzog, Cohen-Macaulay Rings, Cambridge University press, Cambridge, 1993.

[3] L. W. Christensen, Gorenstein Dimensions, Lecture Notes in Math. Vol. 1747, Springer, Berlin, 2000. 
[4] L. W. Christensen - H. B. Foxby - H. Holm, Beyond Totally Reflexive Modules and Back: a survey on Gorenstein dimensions, "Commutative Algebra-Noetherian and non-Noetherian Perspectives”, 101-143, Springer, New York, 2011.

[5] M. D’Anna, A construction of Gorenstein rings, J. Algebra 306 (2006), pp. 507-519.

[6] M. D'Anna - M. Fontana, An amalgamated duplication of a ring along an ideal, J. Algebra Appl. 6, no. 3 (2007), pp. 443-459.

[7] E. E. Enochs - O. M. G. JEndA, Relative Homological Algebra, de Gruyter Expositions in Mathematics, vol. 30, Walter de Gruyter and Co., Berlin, 2000.

[8] N. Epstein - Y. Yao, Criteria for Flatness and Injectivity, Math. Z. 271, no. 3-4 (2012), pp. 1193-1210.

[9] H. B. FoxBy, Gorenstein Modules and Related Modules, Math. Scand. 31, (1972), pp. 267-284.

[10] E. S. GoLoD, G-dimension and Generalized Perfect Ideals, Trudy Mat. Inst. Steklov. 165, (1984), pp. 62-66.

[11] H. Holm - P. Jørgensen, Cohen-Macaulay Homological Dimensions, Rend. Semin. Mat. Univ. Padova, 117 (2007), pp. 87-112.

[12] M. ChHiti - N. MAhdou, Homological dimension of the amalgamated duplication of a ring along a pure ideal, Afr. Diaspora J. Math. (N. S.) 10, no. 1, (2010), pp. 1-6.

[13] N. Mahdou - M. Tamekkante, Gorenstein global dimension of an amalgamated duplication of a coherent ring along an ideal, Mediterr. J. Math. 8, no. 3 (2011), pp. 293-305.

[14] M. NaGATA, Local Rings , Interscience, New York, 1962.

[15] S. Sather-WaGstafF, Semidualizing modules, URL:http://www.ndsu.edu/ pubweb/ ssatherw/.

[16] J. SHAPIRO, On a construction of Gorenstein rings proposed by M. D'Anna, J. Algebra, 323 (2010), pp. 1155-1158.

[17] E. Tavasoli - M. Salimi - A. Tehranian, Amalgamated duplication of some special rings, Bulletin of Korean Math. Soc. 49 no. 5 (2012) pp. 989-996.

[18] W. V. Vasconcelos, Divisor Theory in Module Categories, North-Holland Math. Stud., vol. 14, North-Holland Publishing Co., Amsterdam, (1974).

[19] T. Wakamatsu, On modules with trivial self-extensions, J. Algebra, 114, no. 1 (1988), pp. 106-114.

[20] S. Yassemi, On flat and injective dimension, Italian Journal of Pure and Applied Mathematics, N. 6 (1999), pp. 33-41.

Manoscritto pervenuto in redazione il 26 Ottobre 2011. 
